\title{
Die Biomasse mariner Makrobenthos-Gesellschaften im Einflußbereich der Klärschlammverklappung vor der Elbemündung
}

\author{
U. Mühlenhardt-Siegel \\ Zoologisches Institut und Museum; Martin-Luther-King-Platz 3, D-2000 Hamburg 13, \\ Bundesrepublik Deutschland
}

\begin{abstract}
Biomass of marine macrofauna communities in a dumping area of sewage sludge near the Elbe estuary. The macrofauna of a dumping area in the eastern part of the German Bight (North Sea) was investigated in July, August and November, 1978 at five stations situated on a transect including central and peripheral areas of the dumping region. Abundance and biomass (ash free dry weight) of the macrofauna and its variation from July to November were analysed as well as the biomass of different taxa. Molluscs dominated over polychaetes, crustaceans and echinoderms. A positive correlation seemed to exist between mud content and biomass at the peripherally situated stations. In the central sewage sludge area, however, the biomass values were reduced. In late autumn the biomass decreased in the entire area due to the death of Diastylis rathkei, Abra alba and Pectinaria koreni. These species were replaced by the mollusc Nucula turgida and polychaete Nephtys hombergii. In autumn the biomass values also showed a distinct minimum at the central stations.
\end{abstract}

\section{EINLEITUNG}

Seit 1961 hat eine Schute ca. jeden zweiten Tag westlich von Feuerschiff "Elbe 1" auf einem Areal von $30 \mathrm{~km}^{2}$ Fläche den Faulschlamm der Hamburger Großkläranlage Köhlbrandhöft verbracht. Dieses Verklappen erfolgte bei zweistündiger Fahrt über das Schraubenwasser. Die monatliche Klärschlammenge betrug im Jahr 1962 ca. $20000 \mathrm{~m}^{3}$ und $197822000 \mathrm{~m}^{3}$. Die Verklappung in diesem Teil der Deutschen Bucht wurde im Juli 1980 zum Teil und im Oktober des gleichen Jahres ganz eingestellt.

Das ausgewählte Areal ist ein natürliches Schlickgebiet der Deutschen Bucht, in welchem durch die Tideströmung eine Verdriftung des Feinmaterials und eine ständige Sauerstoffversorgung gewährleistet sind. Es erschien deshalb für eine Verbringung geeignet, weil das dort lagernde Sediment in seiner Konsistenz dem Klärschlamm ähnelte. Es stellte sich die Frage, ob die Verklappung des Klärschlammes die Bodenfauna nachweislich beeinflußt.

In der Deutschen Bucht untersuchte Caspers (1978) die Artenzusammensetzung, und Rachor (1977, 1980) analysierte die Faunenverarmung des Makrobenthos im Rahmen eines Langzeitprogramms. In der vorliegenden Arbeit soll besonders die Biomasse berücksichtigt werden, weil sie genauere Aussagen über die Produktion zuläßt als die Angabe von Arten- und Individuenzahlen. So kann trotz geringer Artenzahl eine hohe 
Biomasse vorliegen, z. B. bedingt durch eine Massenentwicklung von Abra alba, wie sie Caspers (1978) für die Jahre 1973-74 beschrieb.

\section{UNTERSUCHUNGSGEBIET UND METHODE}

Fünf Stationen, die auf einem Nord-Südquerschnitt im Verklappungsgebiet liegen, wurden ausgewählt (Abb. 1, Tab. 1).

Die Stationen 1 und 2 hatten ein ähnliches Sediment, bestehend aus festem, kleiigem Schlick mit Feinsandeinlagerungen und Schill, während die Stationen 3 und 4 weichen, stark nach $\mathrm{H}_{2} \mathrm{~S}$ riechenden Schlick mit einer geringen Oxidationsschicht $(<2)$ $\mathrm{mm}$, aufwiesen. Das Sediment der Station 5 bestand aus Schlick mit Feinsandeinlagerungen, dem aber die kleiige Komponente fehlte. Die Probennahmen erfolgten im Sommer (Juli), Spätsommer (August) und im Herbst (November) 1978.

Nach Stripp (1969) reichen zur Erfassung von $90 \%$ der Artenzahl 8 Parallelproben mit einer Fläche von $0,1 \mathrm{~m}^{2}\left(=0,8 \mathrm{~m}^{2}\right)$. In der vorliegenden Arbeit wurden sogar 5 Parallelproben mit einem van Veen-Backengreifer der Fläche $0,2 \mathrm{~m}^{2}\left(=1,0 \mathrm{~m}^{2}\right)$ an jeweils einer Station genommen; somit dürften über $90 \%$ der Arten erfaßt worden sein. Auch nach Lie (1968) reichen 5 Parallelproben an jeder Station aus für eine adäquate Erfassung der biologischen Daten und ihrer Absicherung.

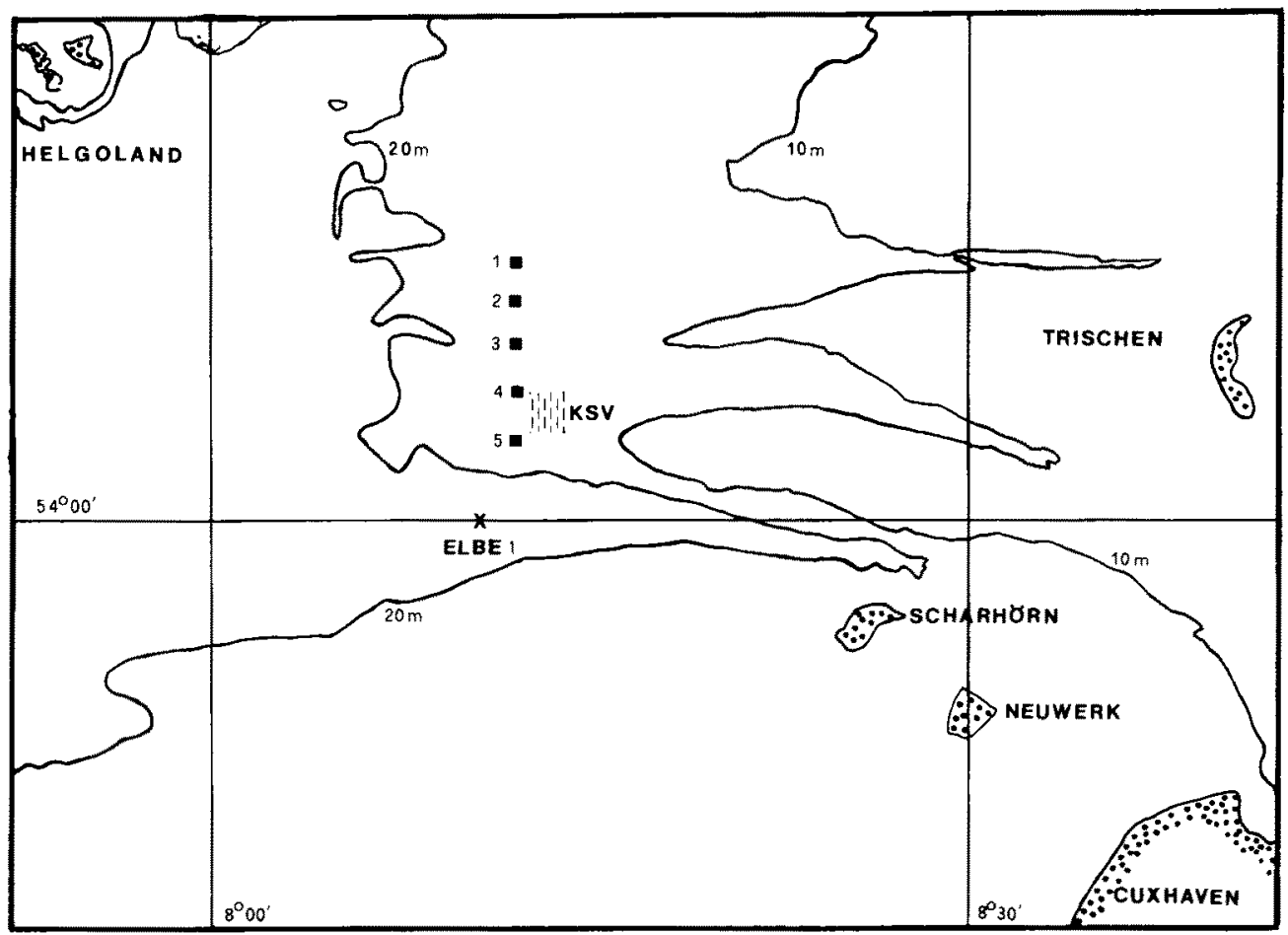

Abb. 1. Lage der 5 Untersuchungsstationen in der Deutschen Bucht (KSV = Gebiet der Einleitung bis 1980) 
Tab. 1. Angaben über Position und Tiefe des Untersuchungsgebietes

\begin{tabular}{|c|c|c|}
\hline Station & Position & Tiefe $(\mathrm{m})$ \\
\hline 1 & $5406,2 \mathrm{~N} / 0812,2 \mathrm{E}$ & $18 \mathrm{~m}$ \\
\hline 2 & $\begin{array}{llll}54 & 05,2 & / 08 & 12,2\end{array}$ & $17 \mathrm{~m}$ \\
\hline 3 & $5404,1 / 0812,2$ & $15 \mathrm{~m}$ \\
\hline 4 & $5403,0 / 0812,3$ & $15 \mathrm{~m}$ \\
\hline 5 & $\begin{array}{llll}5401,9 & / 08 & 12,3\end{array}$ & $16 \mathrm{~m}$ \\
\hline
\end{tabular}

Jede der 5 Parallelproben wurde an Bord mit einem Sieb der Maschenweite 0,5 mm ausgesucht und bis zur weiteren Bearbeitung in Formaldehyd fixiert, das mit Süßwasser auf $4-5 \%$ verdünnt und mit Urotropin gepuffert war.

Da die fünf Parallelproben einer Station getrennt bearbeitet wurden, liegen pro Station und Monat fünf Werte vor. Angegeben werden hier das arithmetische Mittel der Parallelproben und die Standardabweichung (s). Die Signifikanz der Unterschiede der ermittelten Biomassewerte wurde mit Hilfe des t-Testes nach Student geprüft (nach vorheriger Anwendung des F-Testes; vgl. Elliot, 1971).

Nach der systematischen Bestimmung wurde die Biomasse als aschefreies Trockengewicht der Benthosgruppen ermittelt. Zunächst wurde das Material $16-18 \mathrm{~h}$ bei $70{ }^{\circ} \mathrm{C}$ bis zur Gewichtskonstanz getrocknet (Brunswig, 1973; Lovegrove, 1962; Lappalainen \& Kangas, 1975). Anschließend erfolgte nach langsamem Aufheizen das Verglühen der organischen Substanz $7 \mathrm{~h}$ bei $510^{\circ} \mathrm{C}$. Diese Methode ähnelt der von Lie (1968) und der von Lappalainen \& Kangas (1975). Bei der Temperatur von $>500^{\circ} \mathrm{C}$ wird alles organische Material verbrannt, ohne daß der Kalk angegriffen wird.

Die Crustaceen Crangon crangon und Portunus holsatus sowie die Mysidacea wurden nicht berücksichtigt, da sie auf Grund ihrer Fluchtreaktion mit dem Bodengreifer nicht quantitativ erfaßt werden konnten.

\section{ERGEBNISSE}

Im Untersuchungsgebiet wurden insgesamt 67 Benthosarten festgestellt, die gemäß ihren Ansprüchen an das Sediment unterschiedlich häufig an den fünf Stationen vorkamen. Bezüglich der Artenzahl dominierten die Polychaeta. Die Crustacea, Mollusca und Echinodermata stellten die übrigen Gruppen. Tabelle 2 zeigt die Verteilung der Arten auf die Stationen. An Hand dieser Artenlisten und der Abbildung 2 erkennt man, daß die verschiedenen Benthosarten nicht gleich häufig an den Stationen vorkommen. Die Stationen 1 und 2, die am nördlichen Rand des Klärschlammgebietes liegen, zeigten in allen drei Monaten eine hohe Artenzahl, während die beiden im zentralen Verklappungsgebiet liegenden Stationen 3 und 4 die geringsten Artenzahlen aufwiesen. Die südliche Station 5, die auf Grund der Tide und durch den Zufluß von Weser und Elbe von Klärschlamm nur gering beeinflußt wurde, zeigte in allen Untersuchungsmonaten eine hohe Artenzahl.

Im Juli waren im gesamten Untersuchungsgebiet 37 Arten zu finden, im August 58 und im November 27 Arten. An der Station 1 waren $62 \%$ dieser Arten im Juli vertreten, im August $64 \%$ und im November $63 \%$. Allgemein ist zu sagen, daß die relative 
Tab. 2. Die Arten im Untersuchungsgebiet sowie Nachweise ihres Vorkommens im Juli (J), August (A) und November (N) 1978

\begin{tabular}{|c|c|c|c|c|c|}
\hline \multirow[b]{2}{*}{ Species } & \multirow[b]{2}{*}{1} & \multicolumn{3}{|c|}{ Stationen } & \multirow[b]{2}{*}{5} \\
\hline & & 2 & 3 & 4 & \\
\hline \multicolumn{6}{|l|}{ Polychaeta } \\
\hline Harmothoe impar (Johnston) & J & $J \mathrm{~N}$ & & & \\
\hline Harmothoe longisetis (Grube) & & & & & $N$ \\
\hline Harmothoe sarsi (Kinberg) & A & $\mathrm{J}$ & & & A \\
\hline Pholoe minuta (Fabricius) & JAN & JA & A & & A \\
\hline Anaitides sp. & JA & $J$ & & & JAN \\
\hline Anaitides subulifera (Eliason) & & & & & JA \\
\hline Anaitides groenlandica (Oersted) & & & & & A \\
\hline Eumida punctifera (Grube) & JA & & & & JA \\
\hline Nereis longissima Johnston & AN & A & & & $\mathrm{J}$ \\
\hline Nephtys hombergii Savigni & JAN & JAN & JAN & JAN & JAN \\
\hline Glycera capitata Oersted & & & & & A \\
\hline Goniada maculata Oersted & & & & & $\mathrm{J}$ \\
\hline Scoloplos armiger (O. F. Müller) & $J$ & JA & & & JAN \\
\hline Poecilochaetus serpens Allen & & & & & A \\
\hline Spio filicornis (O. F. Müller) & A & & & & JA \\
\hline Polydora ligni Webster & & A & & & \\
\hline Polydora pulchra Carazzi & & & & & A \\
\hline Spiophanes bombyx (Claparède) & A & & & & JA \\
\hline Magelone papillicornis F. Müller & A & & & & JA \\
\hline Scalibregma inflatum Rathke & A & A & $\mathrm{J}$ & & A \\
\hline Ophelina acumenata Oersted & & & & & $\mathrm{J}$ \\
\hline Capitella capitata (Fabricius) & $\mathrm{J}$ & & & & $\mathrm{J}$ \\
\hline Owenia fusiformis Delle Chiaje & $\mathrm{J}$ & & & & A \\
\hline Pectinaria koreni Malmgren & JAN & JA & JAN & $\mathrm{JA}$ & JAN \\
\hline Ampharete sp. Malmgren & A & AN & & & \\
\hline Lanice conchilega Pallas & JAN & JA & J & $\mathrm{J}$ & JAN \\
\hline \multicolumn{6}{|l|}{ Mollusca } \\
\hline $\begin{array}{l}\text { Nucula turgida Leckenby \& Marshall } \\
\text { Mytilus edulis Linné }\end{array}$ & JAN & $\begin{array}{l}\text { JAN } \\
\text { A }\end{array}$ & JAN & JAN & JAN \\
\hline Montacuta ferruginosa (Montagu) & A & & & & \\
\hline Mysella bidentata (Montagu) & JAN & JAN & AN & $\mathrm{J}$ & JAN \\
\hline Cardium edule Linné & & A & & A & \\
\hline Venerupis pullastra (Montagu) & & & & & A \\
\hline Venus gallina Linné & & & & & A \\
\hline Petricola pholadiformis Lamarck & JA & A & & & \\
\hline Mactra corallina (Linné) & & & & & $\mathrm{J}$ \\
\hline Spisula subtruncata Da Costa & AN & A & & & \\
\hline Macoma baltica (Linné) & JAN & JAN & JAN & $J N$ & JAN \\
\hline Angulus fabula Gmelin & & & & & AN \\
\hline Abra alba (S. Wood) & JAN & JAN & JAN & JA & JAN \\
\hline Phaxas pellucidus (Pennant) & & & & & $J$ \\
\hline Corbula gibba (Olivi) & $J N$ & & & & \\
\hline Thracia phaseolina & & & & & $J$ \\
\hline Buccinum undatum Linné & A & & & & \\
\hline
\end{tabular}


Tab. 2. Fortsetzung

\begin{tabular}{|c|c|c|c|c|c|}
\hline \multirow[b]{2}{*}{ Species } & \multicolumn{5}{|c|}{ Stationen } \\
\hline & 1 & 2 & 3 & 4 & 5 \\
\hline \multicolumn{6}{|l|}{ Crustacea } \\
\hline Gammarus sp. & AN & A & & & A \\
\hline Melita obtusa (Montagu) & A & & & & \\
\hline Nototropis swammerdami (Milne Edwards) & JAN & A & & A & AN \\
\hline Nototropis falcatus (Metzger) & & A & & & \\
\hline Orchomenella nana (Köyer) & & & & & A \\
\hline Amphilochus manudens Bate & & & & & A \\
\hline Ampelisca brevicornis (A. Costa) & & & & & A \\
\hline Bathyporeia guilliamsoniana (Bate) & A & & & & $\mathrm{N}$ \\
\hline Perioculodes longimanus (Bate \& Westwood) & & & & & A \\
\hline Pontocrates altamarinus (Bate \& Westwood) & & & & & $\mathrm{N}$ \\
\hline Photis longicaudata (Bate \& Westwood) & JA & A & & & A \\
\hline Microprotopus maculatus Norman & A & A & & & A \\
\hline Dulichia monocantha Metzger & & & & & A \\
\hline Pariambus typicus (Kröyer) & A & $\mathrm{J}$ & A & & A \\
\hline Paramysis kervillei (G. O. Sars) & $A N$ & $\mathrm{~N}$ & & $N$ & AN \\
\hline Bodotria scorpioides (Montagu) & A & & & & A \\
\hline Pseudocuma longicornis (Sp. Bate) & $\mathrm{A}$ & & & & \\
\hline Diastylis rathkei (Kröyer) & JAN & JAN & JAN & JAN & JAN \\
\hline Crangon crangon Linné & JAN & JAN & JA & JAN & JAN \\
\hline Eupagurus bernhardus Linné & JA & A & & & \\
\hline Corystes cassivelaunus (Pennant) & & & & & A \\
\hline Portunus holsatus Fabricius & JA & A & & & JA \\
\hline \multicolumn{6}{|l|}{ Echinodermata } \\
\hline Asterias rubens Linné & & $J$ & & & \\
\hline Ophiura texturata Lamarck & JAN & JAN & JAN & JAN & JA \\
\hline Echinocardium cordatum (Pennant) & $\mathrm{A}$ & & & & \\
\hline
\end{tabular}

Artenzahl an dieser Station konstant war. Bei der Station 2 waren im Juli nur $43 \%$ aller Arten aufzufinden, im August $48 \%$ und im November nur noch $40 \%$. Diese Station zeigte also keine so große Artenvielzahl wie die Station 1. Deutlich weniger Arten waren an der Station 3 anzutreffen: im Juli $27 \%$, im August $19 \%$ und im November $30 \%$ aller Arten.

Die Station 4 hatte auch sehr wenig Arten aufzuweisen: Im Juli $30 \%$, im August $16 \%$ und im November $26 \%$. Ganz anders lagen die Verhältnisse an der Station 5 vor, an der im Juli $73 \%$ im August $74 \%$ und im November $78 \%$ aller Arten zu finden waren.

Welche Arten an den Klärschlammstationen nicht vorkamen, ist der Artenliste zu entnehmen (Tab. 2). Allgemein kann man erkennen, daß in den Klärschlammstationen von den Polychaeta außer Nephtys hombergii, Pectinaria koreni, Lanice conchilega, Scalibregma inflatum, Pholoë minuta und Eteone longa alle anderen Arten fehlten. Von den Mollusca kamen nur Nucula turgida, Mysella bidentata, Cardium edule, Macoma baltica und Abra alba vor. Diastylis rathkei, Crangon crangon, Paramysis kervillei und Nototropis swammerdami sind die einzigen Crustacea, die an dieser Station auftraten. Es fällt das Fehlen der Amphipoda - mit der einzigen Ausnahme Nototropis swammer- 
dami - auf. Dieses Ergebnis deckt sich mit dem von Pearce (1972). Von den Echinodermata war Ophiura texturata einziger Vertreter im Klärschlammgebiet.

Diese Ergebnisse sagen allerdings nichts über die Biomasse dieser Stationen aus. Betrachtet man die Verteilung der Gesamtbiomassen an den Untersuchungsstationen (Abb. 3), so erkennt man, daß eine ähnliche Verteilung wie für die Artenzahl (Abb. 2) vorliegt. Im folgenden werden die fünf Stationen bezüglich der Anteile einzelner Organismengruppen an der Biomasse analysiert (Abb. 4).

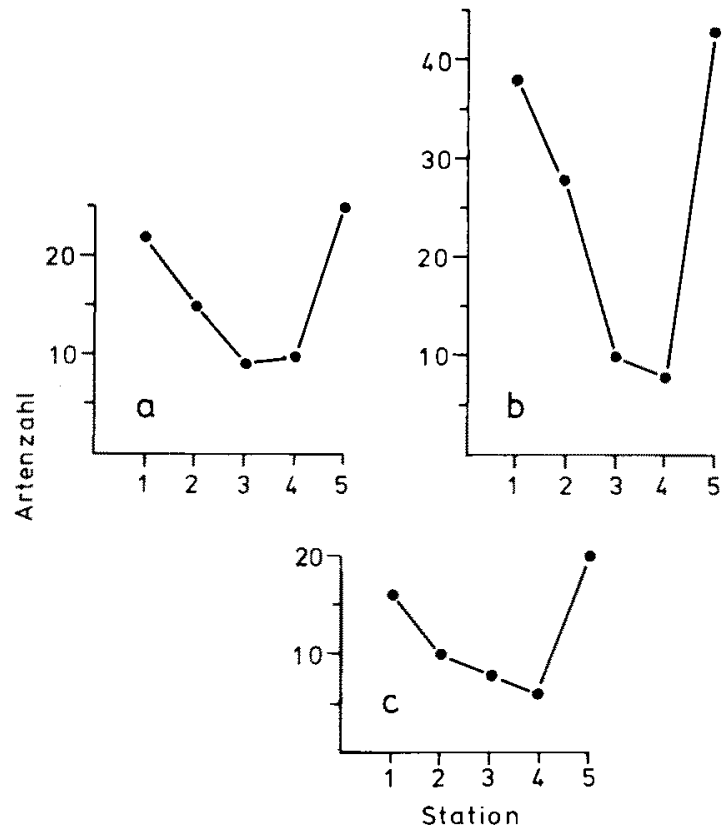

Abb. 2. Häufigkeit der Artenzahlen auf den Untersuchungsstationen in den Monaten Juli (a), August (b) und November (c) pro $\mathrm{m}^{2}$

Station 1. Das Gewicht der organischen Substanz schwankte je nach Untersuchungszeit zwischen $3,5 \mathrm{~g} / \mathrm{m}^{2}$ ( $\left.\mathrm{s}=1,9\right)$ und $5,5 \mathrm{~g} / \mathrm{m}^{2}(\mathrm{~s}=2,3)$. Den Hauptanteil stellten in allen Proben die Mollusca, vertreten vor allem durch Nucula turgida. Die zweitstärkste Gruppe waren die Polychaeta mit Nephtys hombergii als häufigste Art. Es folgen die Echinodermata, allein repräsentiert durch Ophiura texturata. Geringsten Anteil an der Gesamtbiomasse dieser Station hatten die Crustacea, unter denen Diastylis rathkei am häufigsten war.

$\mathrm{St}$ a ti on 2. Diese Station wies im Juli mit $19 \mathrm{~g} / \mathrm{m}^{2}(\mathrm{~s}=10,1)$ die höchste Biomasse aller Stationen auf. Aber auch zu den anderen Jahreszeiten lagen hohe Werte vor: $8,1 \mathrm{~g} /$ $\mathrm{m}^{2}(\mathrm{~s}=4,8)$ im August und $4,6 \mathrm{~g} / \mathrm{m}^{2}(\mathrm{~s}=1,5)$ im November.

Ebenso wie an der vorher genannten Station dominierten die Mollusca (hier ebenfalls hauptsächlich vertreten durch Nucula turgida), an zweiter Stelle folgten die Polychaeta. Auch hier übertraf Nephtys hombergii gewichtsmäßig die anderen häufig auftretenden Arten Lanice conchilega und Pectinaria koreni. Die Crustacea und Echinodermata waren ungefähr gleich stark vertreten. 
Station 3. Außer im Juli, als diese Station eine relativ hohe Biomasse von $5,9 \mathrm{~g} /$ $\mathrm{m}^{2}(\mathrm{~s}=1,9)$ aufwies, zeigte sich an dieser Station eine starke Abnahme der Biomasse in den anderen Monaten: $1,8 \mathrm{~g} / \mathrm{m}^{2}(\mathrm{~s}=1,2)$ im August und $1,9 \mathrm{~g} / \mathrm{m}^{2}(\mathrm{~s}=0,4) \mathrm{im}$ November. Im Juli dominierten auch hier die Mollusca, vertreten durch Nucula turgida, die aber in den folgenden Monaten vergleichsweise zurücktraten. Die anderen drei Gruppen wiesen eine relativ geringere Abnahme auf als die Mollusca. Im November bewirkten nur letztere den leichten Anstieg in der Gesamtbiomasse. Die drei anderen Gruppen sind hauptsächlich durch je eine Art repräsentiert: Pectinaria koreni, Diastylis rathkei und Ophiura texturata.

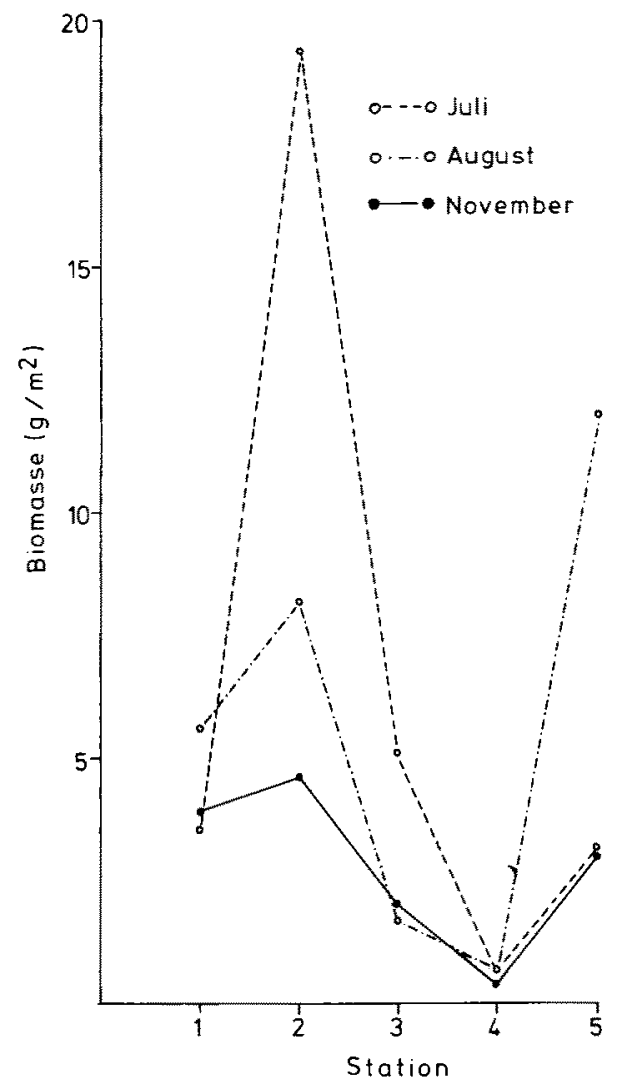

Abb. 3. Biomasse (in $\mathrm{g} / \mathrm{m}^{2}$ ) an den Untersuchungsstationen im Juli, August und November 1978. Mittelwerte aus jeweils fünf Proben

Station 4. Der eben genannte Trend fand sein Pessimum an der Station 4. Die Werte der Biomasse lagen hier nur zwischen $0,4 \mathrm{~g} / \mathrm{m}^{2}(\mathrm{~s}=0,2)$ und $0,5 \mathrm{~g} / \mathrm{m}^{2}(\mathrm{~s}=0,4)$. Im Juli verteilte sich diese geringe Biomasse gleichmäßig auf Mollusca, Polychaeta und Crustacea, während Echinodermata kaum vertreten waren. Auffallend ist, daß unter den Mollusca Abra alba einen größeren Anteil hatte als Nucula turgida. Im August nahm die Biomasse der Echinodermata geringfügig zu. Diese Tendenz hielt bis zum November an, verursacht durch heranwachsende kleine Ophiura texturata; die Biomasse der anderen drei Gruppen war dann besonders gering. 


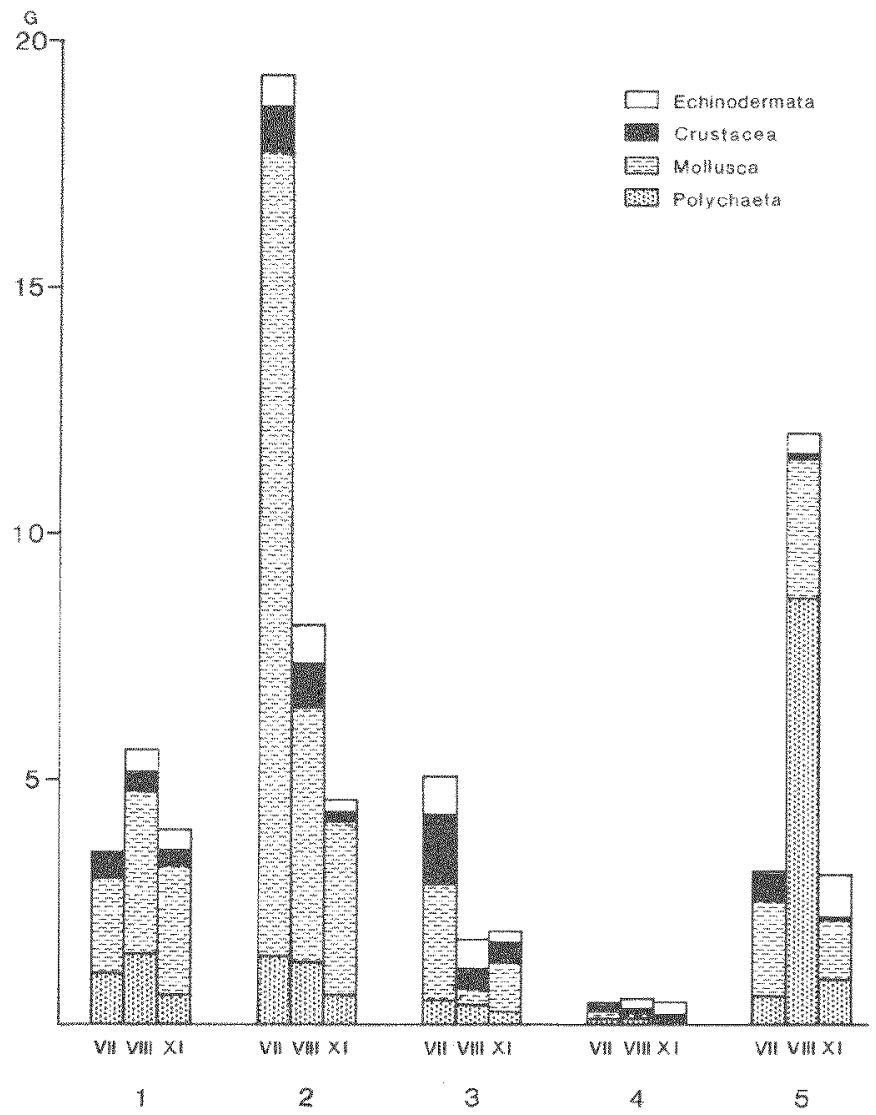

Abb. 4. Vertelung der Biomasse (in $\mathrm{g} / \mathrm{m}^{2}$ ) auf die einzelnen systematischen Gruppen an den Untersuchungsstationen im JuL (VIT). August (VTI) und November (XI) 1979

Station 5. Die sudlichste Station befand sich auf Grund der Strömungsverhältnisse vermuthich nicht mehr im Einflußbereich des Kärschammgebietes. Fier bestand das Benthos uberwiegend aus Molusca mit den Hauptarten Abra aba und Nucula turgida. Im Juli waren die polychaeta und Crustacea ungefahr gleich stark vertreten, während auf die Echinodemata nur der geringste Anteil der Biomasse entiel. Bedingt durch eine ausgedehnte Lanice-Population im August stieg die Biomasse der Polychaeta zu dieser Zeit stark an und war mit $8,7 \mathrm{~g} / \mathrm{m}^{2}(\mathrm{~s}=0,9)$ die zweithöchste einer einzelnen Ant während der gesamten Probennahme. Diese Lanice-Population war im November nicht mehr aufzufinden, so dab die Polychaeta dam wieder in der Gesamtbiomasse nach den Mollusca (Abra alba und Nucula turgida) rangierten.

Die mittlere Biomasse der Station $5 \mathrm{Iag}$ im August signifikant höher als die der tubrigen Stationen, während die der Station 4 im Juli und August signifikant niedriger lag als die Biomasse der benachbarten Stationen 3 und $5(P<0,05)$. Zwischen den Stationen am Rand des Klärschlanmgebietes sind nur zum Teil gesicherte Unterschiede nachzuweisen. Die Biomassewerte der Station 1 in August und die der Station 2 im November waren signifikant höher als die der Station 3. Einen Anstieg der Biomasse 
zum Herbst hin, gefolgt von einer Abnahme bis zum Frühjahr, wie Abb. 4 vermuten läßt und auch Stripp (1969) erkannte, läßt sich aus den vorliegenden Ergebnissen nicht mit Sicherheit feststellen. Ein signifikanter Unterschied $(P<0,05)$ der Biomassewerte im Verlauf der Monate läßt sich nur bei der Station 5 nachweisen. Hier lag der Wert für August höher als der für Juli und November.

\section{DISKUSSION}

Das Untersuchungsgebiet gehört nach Hagmeier (1925) zur Scrobicularia-albaGemeinschaft, die auf Grund ihrer Nähe zum Elbe-Ästuar durch große Nährstoffzufuhr und ein schlickiges Sediment charakterisiert ist.

Die vorliegenden Ergebnisse bestätigen Angaben Remanes (1940), der für dieses Weichbodengebiet eine reiche Besiedlung und daraus resultierend einen hohen Gehalt an Biomasse annimmt, wobei er Faulschlamm ausschließt. Stripp (1969) vergleicht seine Untersuchungen in der Deutschen Bucht mit denen von Kühlmorgen-Hille (1963) in der Kieler Bucht und hält einen Vergleich der Abra-Gemeinschaft aus beiden Gebieten für zulässig. Arntz \& Brunswig (1975) geben für die Abra-Gemeinschaft der Kieler Bucht eine mittlere organische Substanz von $7,9 \mathrm{~g} / \mathrm{m}^{2}$ an, die höher ist als im gesamten Klärschlamm-Untersuchungsgebiet, welche (über drei Monate gemittelt) $4,9 \mathrm{~g} / \mathrm{m}^{2}$ beträgt. Der Anteil der verschiedenen Taxa an der Gesamtbiomasse ist in der AbraGemeinschaft der Kieler Bucht (Arntz \& Brunswig, 1975) ähnlich dem des Untersuchungsgebietes aus der vorliegenden Arbeit. Wie allerdings Caspers (1978) herausstellt, wiesen einige Bereiche des zentralen Klärschlammgebietes in den Jahren 1973 und 1974 bei reduzierter Artenzahl eine beträchtliche Individuenzahl und damit eine hohe Biomasse auf, hauptsächlich bedingt durch eine Massenentwicklung von Abra alba.

Ähnliche Probleme liegen auch in anderen Meeresgebieten vor. So weisen Pearce (1972) und Botton (1979) auf eine Artenarmut in den belasteten Gebieten der New Yorker Bucht hin, wobei beide Autoren eine besondere Empfindlichkeit der Amphipoda gegenüber Klärschlamm andeuten. Diese Tendenz läßt sich auch in den untersuchten Gebieten erkennen (Tab. 2). Smyth (1968) begründet die Artenarmut eines belasteten Meeresgebietes in britischen Gewässern mit einer negativen Beeinflussung der Freßoder Atemmechanismen von Bodentieren durch Abwassereinleitungen. Reish (1980) ermittelte in stark belasteten Gewässern vor Los Angeles eine Artenabnahme, aber eine Zunahme der Individuenzahlen und damit auch der Biomasse. Die hier untersuchten Stationen zeigen jedoch keine vergleichbaren Massenentwicklungen.

Bezüglich der Artenzahl im Untersuchungsgebiet ist deutlich ein Minimum an den zentralen Klärschlammstationen zu erkennen (Abb. 2), was auf einseitige Lebensbedingungen (starke Belastung des Systems durch $\mathrm{H}_{2} \mathrm{~S}$-Bildung oder besonders weiches Substrat) hinweist. An den Randstationen liegen die Artenzahlen bedeutend höher, was den Schluß nahelegt, daß hier ein geringerer Einfluß durch den Klärschlamm vorliegt.

Es ist denkbar, daß gewisse Unterschiede zwischen den Stationen und Monaten vorhanden sind, sie aber durch die starke Streuung der Einzelproben nicht nachweisbar waren. Diese Streuung ist sicherlich - neben der Interpretation als "patchiness" - auch auf die Methode der Probennahme zurückzuführen (Stripp, 1969; Rogal, 1978).

Wie schon oben erwähnt, läßt sich durch fünf Greiferproben zwar die Artenzahl einer Station zu $90 \%$ erfassen (Stripp, 1969), hingegen liefern bei der Berechnung der 
Biomasse von fünf Parallelfängen aus einer Station infolge starker Streuung der Einzelproben nur gemittelte Werte Aussagen über die vorliegenden Verhältnisse. Eine gröBere Genauigkeit der Angabe über die Biomasse könnte durch erhöhte Probenzahl erreicht werden, wobei allerdings zu berücksichtigen ist, daß mit der Decca-Navigation des jeweiligen Forschungsschiffes nur im Bereich von $50 \mathrm{~m}$ eine Position angesteuert werden kann. Beachtet man aber, daß die Benthosfauna auf schon kleinen Räumen sich ändern kann, würde meines Erachtens nur eine Probennahme durch Tauchen an mit Bojen markierten Positionen eine exaktere Auskunft über die Verhältnisse der Biomasseverteilung geben.

Bezüglich der Biomasse liegt die Station 4 signifikant niedriger als die der anderen Stationen, an der Station 3 ist die Wirkung des Klärschlamms an der verringerten Artenzahl zu erkennen. Der Einfluß des Klärschlamms auf die Bodenfauna könnte neben der $\mathrm{H}_{2} \mathrm{~S}$-Bildung auch in seiner Konsistenz liegen. Schon Lie (1969) sowie Lie \& Kisker (1970) geben eine Beziehung zwischen Sedimenttyp und Biomasse an. Wahrscheinlich ermöglicht das weiche Sediment der Stationen 3 und 4 im Untersuchungsgebiet nur wenigen Arten eine Besiedlung. Diese Möglichkeit muß in nachfolgenden Arbeiten durch Sedimentanalysen des Klärschlammgebietes überprüft werden.

Zusammenfassend ist zu sagen, daß sowohl für die Biomasse als auch für die Artenzahl an den Klärschlammstationen deutlich niedrigere Werte gefunden wurden als an den Randstationen. Man kann also nicht nur durch die Reduzierung der Artenzahl, sondern auch an Hand der Biomasseangaben die Belastung dieses Systems erkennen.

Danksagungen. Mein Dank gilt Herrn Prof. Dr. H. Caspers (Hamburg) für die Anregung zur vorliegenden Untersuchung sowie Herrn Dr. E. Rachor und Mitarbeitern (Bremerhaven) für die Mithilfe beim Sammeln des umfangreichen Probenmaterials. Herrn Prof. Dr. C.-D. Zander möchte ich für die Durchsicht des Manuskripts herzlich danken.

\section{ZITIERTE LITERATUR}

Arntz, W. E. \& Brunswig, D., 1975. An approach to estimating the production of macrobenthos and demersal fish in a western Baltic Abra alba community. - Merentutkimuslait. Julk. 239, $195-205$.

Botton, M. L., 1979. Effects of sewage sludge on the benthic invertebrate community of the inshore New York Bight. - Estuar. coast. mar. Sci. 8, 169-180.

Brunswig, D., 1973. Der Nahrungswert makrobenthischer Fischnährtiere der Kieler Bucht im Jahresgang. Diplomarbeit, Univ. Kiel, 65 pp.

Caspers, H., 1978. Ecological effects of sewage sludge on benthic fauna off German North Sea coast. In: Proceedings of the 8th international conference on water pollution research, Sydney 1976. Ed. by S. H. Jenkins. Pergamon Press, Oxford, 951-956.

Elliott, J. M., 1971. Some statistical analysis of samples of benthic invertebrates. - Scient. Publ. Freshwat. Biol. Ass. 25, 1-148.

Hagmeier, A., 1925. Vorläufiger Bericht über die vorbereitenden Untersuchungen der Bodenfauna der Deutschen Bucht mit dem Petersen-Bodengreifer. - Ber, dt. Kommn. Meeresforsch. (N. F.) $1_{t}$ $247-272$.

Kühlmorgen-Hille, G., 1963. Quantitative Untersuchungen der Bodenfauna in der Kieler Bucht und ihre jahreszeitlichen Veränderungen. - Kieler Meeresforsch. 19, 42-66.

Lappalainen, A. \& Kangas, P., 1975. Littoral benthos of the northern Baltic Sea. 11. Interrelationship of wet, dry and ash-free dry weights of macrofauna in the Tvärminne area. - Int. Revue ges. Hydrobiol. $60,297-312$. 
Lie, U, 1968. A quantitative study of benthic infauna in Puget Sound, Washington, USA in 1963-1964. - FiskDir. Skr. (Havunders) 14, $229-556$.

Lie, U., 1969. Standing crop of benthic infauna in Puget Sound and off the coast of Washington. - J. Fish. Res. Bd Can, 26, 55-62.

Lie, U. \& Kisker, D. S., 1970. Species composition and structure of benthic infauna communities off the coast of Washington. - J. Fish. Res. Bd. Can. 27, 2273-2286.

Lovegrove, T., 1962. The effects of various factors on dry weight values. - Rapp. P.-v. Réun, Cons. perm. int. Explor. Mer 153,86-91.

Pearce, J. B., 1972. The effects of solid waste disposal on benthic communities in the New York Bight. In: Marine pollution and sea life. Ed. by M. Ruivo. Fishing News, London, 404-411.

Rachor, E., 1977. Faunenverarmung in einem Schlickgebiet in der Nähe Helgolands. - Helgoländer wiss. Meeresunters. 30,633-651.

Rachor, E., 1980. The inner German Bight - an ecologically sensitive area as indicated by the bottom fauna. - Helgoländer Meeresunters. 33, 522-530.

Reish, D. J., 1980. Effects of domestic wastes on the benthic marine communities of southern California. - Helgoländer Meeresunters. 33, 377-383.

Remane, A., 1940. Einführung in die zoologische Ökologie der Nord- und Ostsee. - Tierwelt Nordu. Ostsee 1a, 1-238.

Rogal, U., Anger, K., Schriever, G. \& Valentin, C., 1978. In-situ investigations on small-scale local and short-term changes of sublittoral macrobenthos in Lübeck Bay. - Helgoländer wiss. Meeresunters. 31, 303-313.

Smyth, J. C., 1968. The fauna of a polluted shore in the Firth of Forth. - Helgoländer wiss. Meeresunters. 17, 216-223.

Stripp, K., 1969. Jahreszeitliche Fluktuationen von der Makrofauna und Meiofauna in der Helgoländer Bucht. - Veröff. Inst. Meeresforsch. Bremerhaven 12, 65-94. 